\title{
Evaluation de la gestion des déchets solides hospitaliers: cas de l'hôpital Jamôt de Yaoundé et de l'hôpital de district de Biyem-Assi
}

\author{
Severin MBOG MBOG ${ }^{1,2 *}$, BIDIAS Jacob ${ }^{2}$, Olivier T SOSSO MAYI ${ }^{3}$, Dieudonne BITONDO ${ }^{1,4}$, \\ Innocent NDOH MBUE ${ }^{1,4 *}$ \\ ${ }^{1}$ Department of Industrial Quality, Hygiene, Safety and Environment, National Advanced School for \\ Engineering, University of Douala, P.O Box 2701, Douala-Cameroon \\ ${ }^{2}$ Department of Plant Biology, Faculty of Sciences, University of Yaoundé I, P.O Box 331 Yaounde- \\ Cameroun \\ ${ }^{3}$ Laboratory of Thermal and Environment, Advanced Teacher's Training College For Technical Education, \\ University of Douala, P.O Box 1872 Douala-Cameroon \\ ${ }^{4}$ Laboratory of Energy, Materials, Modeling and Methods, National Advanced school for Engineering, \\ University of Douala, P.O Box 2701, Douala-Cameroon. \\ *corresponding author severinmbog.sm@gmail.com
}

\section{Résumé}

L’objectif général de cet étude est d'améliorer la gestion des déchets solides au sein de l'Hôpital de district de Biyem-Assi et à l'hôpital Jamôt de Yaoundé.

La démarche méthodologique menée de juillet 2012 à septembre 2013e adoptée, a consisté à des entretiens et des observations directes et l'administration des questionnaires semi-structurés. Cette méthodologie a permis de réaliser l'étude en trois phases la recherche documentaire, la collecte et le traitement des données:

Les résultats obtenus font ressortir deux grandes catégories de déchets : les déchets assimilables aux ordures ménagères (DAOM); les déchets d'activités de soins à risques (DASRI), 24,46 \% est assimilable aux (DAOM) et 75,54 \% (DASRI) à l'Hôpital Jamôt de Yaoundé. Tandis qu'à l'Hôpital de District de Biyem-Assi 86,23 \% est assimilable aux (DAOM) et 13,77\% (DASRI). Par contre l'appréciation de l'efficacité du système de gestion des déchets solides révèle qu'à l'HJY après des interviews démontre que le système est mauvais: tandis qu'à l'HDB, le système est plus pris en charge par le personnel.

$\mathrm{Au}$ vu des observations et constats faites, une amélioration du système de gestion des déchets solides et l'accroissement de la sensibilisation du personnel et des usagers sur les modes de gestion des déchets solides s'imposent.

Mots clés. Déchets solides, gestion, environnement, hôpital Jamôt de Yaoundé, hôpital de District de Biyem-Assi.

Received: 14/02/2020

Accepted: $12 / 07 / 2020$

DOI: https//dx.doi.org/10.4314/jcas.v16i2.1

(C) The Authors. This work is published under the Creative Commons Attribution 4.0 International Licence. 


\begin{abstract}
The general objective of this study is to improve the management of the solid waste within Biyem-Assi District Hospital and the Jamôt Hospital, Yaoundé.

The work was carried out from July 2012 to September 2013, and consisted to interviews and the direct observations and the administration of semi-structured questionnaires. This methodology permitted us to achieve the survey in three phases: the documentary research, the collection and the treatment of the data:

The results obtained revealed two big categories of garbage taken out again: the garbage assimilated to the household waste $(\mathrm{HhW})$; hospital waste (HoW). About 24, $46 \%$ was assimilated to (HhW) and 75, $54 \%(\mathrm{HoW})$ in the Yaounde Jamôt Hospital. While to the hospital of District of Biyem-Assi 86, $23 \%$ is assimilated to them $(\mathrm{HhW})$ and $13,77 \%(\mathrm{DHoW})$. On the other hand the appreciation of the efficiency of the system of management of the solid waste reveals that to the HJY after interviews demonstrates that the system is bad: while to the HDB, the system is taken more in charge by the staff.

Within sight of the observations and reports made, an improvement of the system of management of the strong garbage and the growth of the sensitization of the staff and users on the fashions of management of the strong garbage impose themselves.
\end{abstract}

Keywords. Solid waste, system of management, environment, Yaoundé Jamôt Hospital , District Hospital of Biyem-Assi. 


\section{Introduction}

La gestion des déchets solides, en général pose des problèmes majeurs auxquels sont confrontées bon nombre de pays notamment ceux en voie de développement. Les quantités importantes de déchets produits par les établissements de santé de même que l'intérêt accru porté à la prévention des infections font de la gestion des déchets solides hospitaliers une problématique qui prend de plus en plus de l'ampleur, surtout si l'on considère la voie du développement durable dans laquelle s'est engagé le Cameroun [12]. Les déchets hospitaliers dangereux représentent un risque professionnel pour la santé du personnel hospitalier et des personnes chargées de leur transport, leur traitement et leur évacuation. Ainsi la production de ces déchets pose deux problèmes majeurs, la responsabilité de leur gestion et le choix des techniques de leur traitement [1-5].

En effet, les principes les plus importants qui gouvernent des programmes efficaces en matière de gestion de déchets hospitaliers sont l'attribution de la responsabilité légale et financière d'une gestion sûre à celui qui génère les déchets, et la mise en œuvre du principe de précaution [2-3]. L'OMS (Organisation Mondial de la Santé) recommande un budget pour la gestion des DSMP (Déchets Solides Médicaux et Pharmaceutiques) au moins de $0,25 \%$ du budget total de fonctionnement de l'hôpital et il doit être prévu dans le cadre du budget d'hygiène de chaque hôpital [6-9]

En matière d'élimination des déchets, les établissements de soins sont concernés à double titre: en tant que producteurs de déchets donc responsables de leur bonne élimination et en tant que acteurs de santé publique soucieux d'une bonne hygiène pour la protection de la population. Kandela souligne que la promotion et la protection de la santé sont inextricablement liées à la promotion et à la protection des droits et de la dignité humaine.
L'hôpital joue un grand rôle dans la protection et la promotion de la santé. Il constitue une véritable zone dans la ville. Pour cela l'hôpital n'est pas un producteur de déchets comme les autres [9].

La gestion des déchets hospitaliers souffre d'un manque relativement important de soutien financier. Il n'existe pas de budget spécifique alloué aux déchets hospitaliers [14]. Selon l'OMS, une gestion adéquate de ces déchets nécessiterait une politique d'hygiène appropriée, des moyens humains, matériels et financiers suffisants, du personnel formé et une réglementation adéquate pour pouvoir prévenir, si non réduire, tout risque pour les patients, les professionnels et l'environnement [10-13]. Manga [18] rapporte que «L'insuffisance des ressources financières limite considérablement les initiatives visant à assurer une gestion correcte des DH (Déchets hospitaliers ): la majorité des formations sanitaires n’a prévu une ligne budgétaire spécifique pour prendre en charge les activités de gestion des $\mathrm{DH}$ » Les principes les plus importants qui gouvernent des programmes efficaces en matière de gestion de déchets hospitaliers sont l'attribution de la responsabilité légale et financière d'une gestion sûre à celui qui génère les déchets, et la mise en œuvre du principe de précaution [14].

Diverses publications et enquêtes ont montré que les conditions actuelles de gestion des déchets solides hospitaliers ne sont pas toujours satisfaisantes. Si cette situation peut se comprendre, cela n'implique pas que l'on ne tente pas d'y remédier. Certaines initiatives menées par le gouvernement permettent d'envisager l'avenir avec un relatif optimisme quant à l'évolution de la situation comme la promulgation du décret No2012/2809/PM du 26 septembre 2012 relatif aux conditions de tri, de collecte, de stockage, de transport, de récupération, de recyclage, de traitement et d'élimination finale des déchets qui stipule à son article 12 (1) que: les déchets 
médicaux et pharmaceutiques font l'objet d'une gestion spécifique visant à éviter toute atteinte à la santé de l'homme.

Malgré la législation qui n'a cessé d'évoluer, il reste beaucoup de chemin à parcourir pour que les impacts des DH sur la santé et l'environnement soient atténués et atteignent un niveau raisonnable, selon les normes internationales déterminées par l'OMS ou le PNUE (Programme des Nations Unies pour l'environnement).

Le présent travail s'inscrit dans ce cadre, il a pour objectif général d'améliorer la gestion des déchets solides à l'Hôpital Jamôt de Yaoundé et à l'Hôpital de District de Biyem-Assi.

Spécifiquement le présent travail se propose de: - faire un état des lieux de la gestion des déchets solides hospitaliers de l'Hôpital Jamôt de Yaoundé et l'Hôpital de District de Biyem-Assi; - évaluer le mode de gestion des déchets solides de l'hôpital Jamôt de Yaoundé et l'Hôpital de District de Biyem-Assi;

- identifier et évaluer les impacts environnementaux et sanitaires des déchets solides de l'hôpital Jamôt de Yaoundé et l'hôpital de district de Biyem-Assi;

- élaborer un plan de gestion de déchets solides hospitaliers à ces deux hôpitaux.

\section{Matériel et méthodologie}

L'état des lieux de la gestion des déchets solides à l'Hôpital Jamôt de Yaoundé et à l'Hôpital de District de Biyem-Assi, a été réalisé grâce aux observations et aux enquêtes réalisés auprès du personnels médicaux, médico-techniques et des usagers lors de nos différentes descentes sur le terrain.

L'enquête a été réalisée auprès du personnel et des usagers de l'HJY et de l'HDB. Une trame d'enquête a été administrée aux personnels de
l'HPJY et de l'HDB, aux usagers et aux personnes environnantes du site d'étude (Annexe 1). Ces enquêtes ont été réalisées suivant les techniques d'enquêtes et de sondage proposés par Brossier et Dussai.

Les entretiens ont été réalisés avec certains intervenants dans la gestion des déchets solides médicaux : le directeur de l'hôpital Jamôt, le médecin chef de l'hôpital de District de BiyemAssi, les surveillants généraux, les majors des services techniques; les agents d'entretien; les agents de collecte les professionnels de santé, les techniciens hygiénistes. Cela nous a permis de clarifier les points d'ombres, de recueillir les problèmes vécus et les points de vue des différents intervenants.

Dans le souci d'avoir des données fiables, les enquêtes et les entretiens ont été complétés par des observations directes et des prises d'images enfin d'illustrer le travail. Ces observations nous ont permis de voir de plus près les pratiques réelles en matière de la gestion des déchets dans les différents hôpitaux, de mieux comprendre l'organisation de la gestion des déchets dans ces hôpitaux et voir si celle-ci est conforme aux normes nationales en matière de gestion des déchets hospitaliers.

La participation au travail de pré collecte et de collecte dans nos deux hôpitaux nous a permis d'identifier ensuite d'évaluer le mode de gestion des déchets solides de nos deux hôpitaux grâce aux observations et aux entretiens directs.

Les données collectées ont été saisies à l'aide d'un logiciel Excel 2013.

\section{Résultats}

\section{Etat de l'environnement du site}

Les descentes effectuées sur le terrain ont permis de constater que la gestion des déchets solides de 
l'Hôpital Jamôt de Yaoundé et de l'Hôpital de District de Biyem-Assi fait face à beaucoup de difficultés.

On a noté des aspects dégradants rendant les hôpitaux insalubres (Fig.1.). Il s'agit :

- local de stockage des DASRI inexistant dans nos deux hôpitaux ;

- bourbiers entravant la circulation;

- mauvais conditionnement;

- de la présence des dépôts d'ordures.

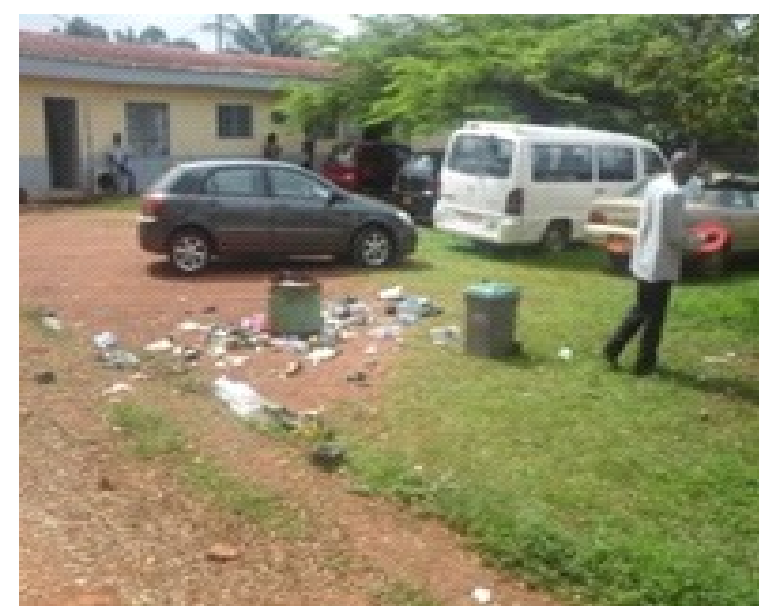

Fig. 1. Dépôt des déchets hospitaliers près des bacs à l'HJY

\section{Etat d'insalubrité}

Les résultats obtenus montrent que 39,22 \% du personnel et des usagers enquêtés affirment que l'Hôpital Jamôt de Yaoundé est moins sale, 28,29 $\%$ trouvent l'Hôpital sale, 19,80 \% trouvent très sale, $5,31 \%$ le jugent propre et 7,38 \% sont restés sans opinion. Tandis que $22,41 \%$ du personnel et des usagers enquêtés affirment que l'Hôpital de District de Biyem-Assi est propre et $26.14 \%$ trouvent l'hôpital sale, 16,53 \% trouvent très sale, $28,33 \%$ le jugent un moins et $6,59 \%$ sont restés sans opinion (Fig.2).

\section{Connaissances du personnel sur les risques liés aux déchets solides hospitaliers}

Chez le personnel des deux hôpitaux (97\%) sont conscients des risques des déchets hospitaliers pour leur santé, un très grand nombre $(93,7 \%)$ pense que leur séparation est utile : ceci prouve que les efforts de sensibilisation sur ce point important ont été efficients.

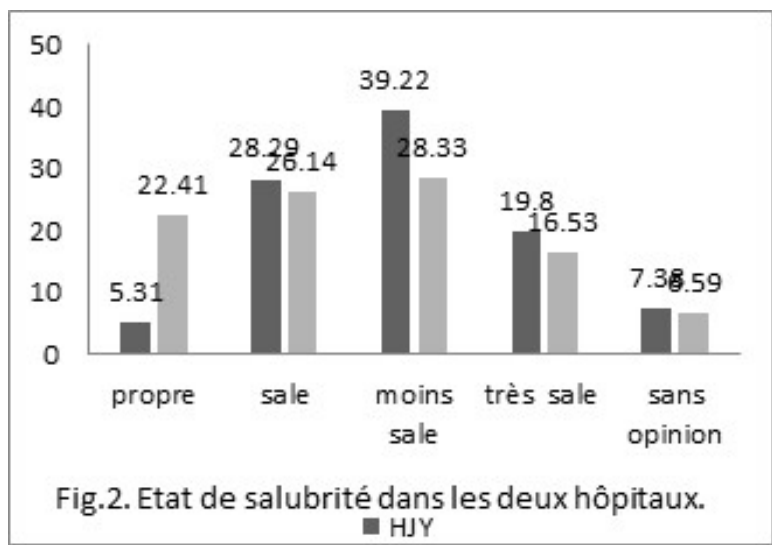

Connaissance de la typologie des déchets solides hospitaliers

Le niveau insuffisant de formation est objectivé, une fois de plus, par les faibles fréquences de personnels connaissant les différents types de déchets produits dans une structure de soin(1,3 $\%$, leur désignation $(4,1 \%)$, et les différentes étapes de la filière de gestion des déchets solides hospitaliers.

Le faible ratio $(27,6 \%)$ de personnels connaissant globalement les codes-couleurs réglementaires, est plus en relation avec le défaut d'information et de diffusion des textes réglementaires. Si la conscience du risque est réelle et acquise pour la quasi-totalité des personnels de santé toutes catégories confondues, il n'en est pas de même pour la formation qui ne ciblé que le quart du personnel.

\section{Connaissances du personnelles sur les causes de la mauvaise gestion des déchets solides hospitaliers}

L'enquête menée sur le terrain auprès du personnel et des usagers sur les causes de la mauvaise gestion des déchets solides hospitaliers révèle à 11,73\% que la mauvaise gestion des déchets solides hospitaliers est due au manque de sensibilisation(A) du personnel et des usagers, à l'insuffisance de ressources financières(B) à 14,28 
$\%$.Tandis que, $8,54 \%$ des personnes interviewées parmi le personnel pensent que cette mauvaise gestion est due à l'insuffisance des ressources matérielles $(\mathrm{C})$ appropriées à la gestion des déchets solides. Par contre 11,98 \% pensent quelle serait due à $(A+B), 17,67 \%(A+C)$ et 15,45\% $(B+C)$. De même, quelques $20,35 \%$ affirment $(A+B+C)$ (Fig.2).

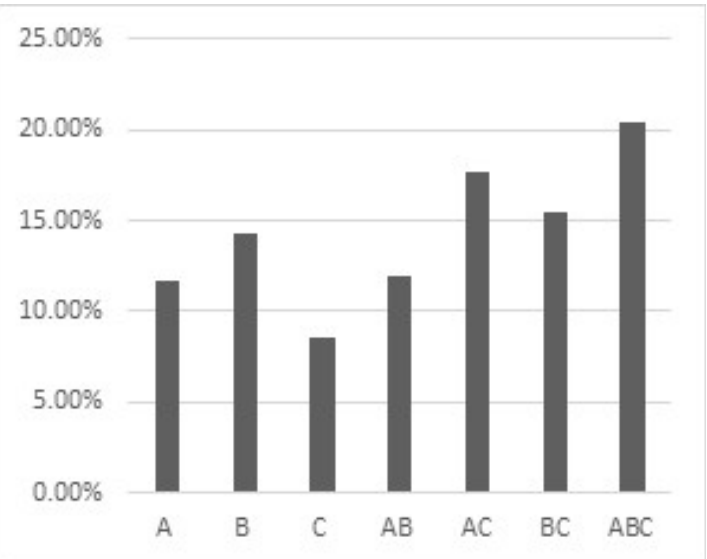

Fig.2.Causes de la mauvaise gestion des déchets solides dans les hôpitaux.Sensibilisation.

(A), Ressources financières(B), Ressources matérielles $(\mathrm{C})$.

\section{Appréciation de l'efficacité du système de gestion des déchets solides}

Les résultats, obtenus pendant les enquêtes réalisées auprès du personnel et des usagers, en rapport à l'appréciation de l'efficacité du système de gestion des déchets solides, relèvent qu'àl'Hôpital Jamôt de Yaoundé seulement 17,36 $\%$ des personnes interviewées parmi le personnel et les usagers pensent que le système de gestion des déchets solides hospitaliers est bon. Par contre $26,77 \%$ le trouvent passable et 55,87\% trouvent qu'il est mauvais. Tandis qu'à l'Hôpital de District de Biyem-Assi 36,43 \% des personnes interviewées parmi le personnel et les usagers le trouvent qu'il est passable et 35,67\%. Par contre le trouvent bon et 27,90\% le trouve qu'il est mauvais (Fig.3.).

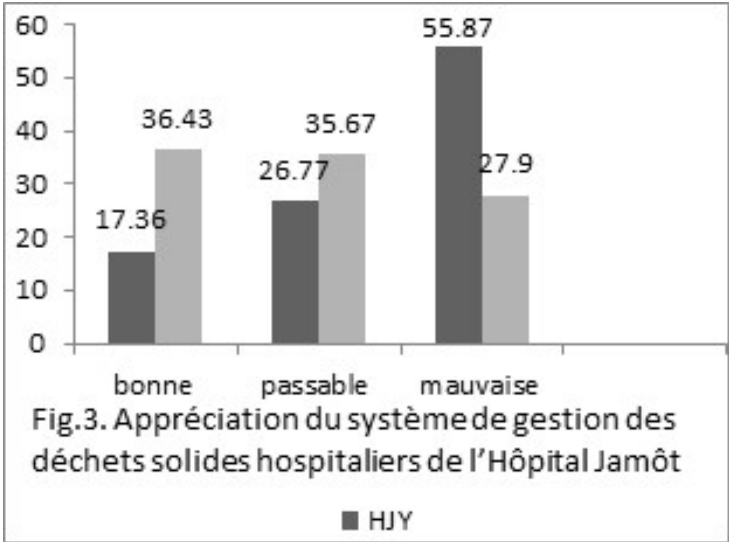

Estimation des déchets produits à l'Hôpital Jamot de Yaoundé et l'Hôpital de District de Biyem-Assi

Les résultats obtenus dans le Tableau I ci-dessous pendant les enquêtes et entretiens réalisées auprès des majors et agents d'entretiens ont permis de regrouper les déchets hospitaliers solides (Hôpital Jamôt de Yaoundé et Hôpital de District de Biyem-Assi) en huit catégories de différentes de matières.

\begin{tabular}{|l|c|l|}
\hline $\begin{array}{l}\text { Matières } \\
\text { hôpitaux }\end{array}$ & HJY(\%) & HDB (\%) \\
\hline Plastiques & 37,23 & 38,18 \\
\hline Papier & 24,37 & 26,14 \\
\hline Carton & 16,11 & 18,66 \\
\hline Verre & 0,7 & 0,5 \\
\hline $\begin{array}{l}\text { Métaux } \\
\text { non ferreux }\end{array}$ & 6,79 & 4,48 \\
\hline $\begin{array}{l}\text { Métaux } \\
\text { ferreux }\end{array}$ & 5,75 & 0,71 \\
\hline Nourriture & 7,64 & 6,47 \\
\hline Divers & 1,41 & 4,86 \\
\hline
\end{tabular}

Organisation du système de gestion des déchets solides hospitaliers

L'hôpital Jamot de Yaoundé procède une unité d'hygiène hospitalière $(\mathrm{UHH})$ en son sein mais ne possède pas de plan clair concernant l'hygiène, il n'existe pas un comité de gestion des déchets. Le personnel responsable de la collecte des DSH à l'intérieur de l'hôpital est composé de deux agents dans la société sous-traitante. L'entretient de chaque pavillon est assuré par un agent de la 
société sous-traitante. La gestion de l'incinérateur est assurée par deux agents. Les DAOM collectés sont stockés dans un bac de $16 \mathrm{~m}^{3}$, tandis que les DASRI sont stockés au niveau de l'incinérateur pour leur élimination directe (Fig.4.). La collecte se fait au niveau de tous les pavillons 1 fois par jour, 5 jours sur 7. Le rythme de la collecte des déchets est irrégulier (les déchets peuvent être collectés 2 jours sur 7, cas du laboratoire et la bronchoscopie ou 3 sur 7 cas du CTA. Ceci est dû au rythme de fonctionnement de chaque service selon les agents. En plus, ils manquent souvent d'équipements de protection appropriés (tenues, gants, masques, des bottes, des tabliers ou blouses de travail, des lunettes de protection etc.). L'évacuation des déchets en dehors de l'hôpital se fait en général par l'intermédiaire des camions par SECA-HYSACAM deux fois par semaine.

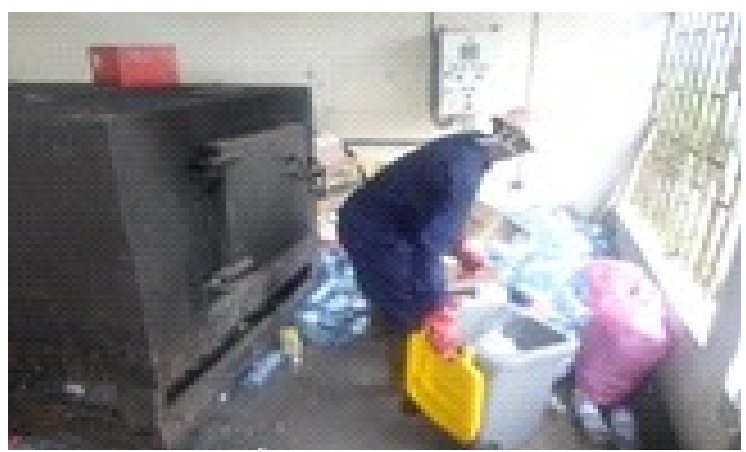

Fig. 4 .Déchets solides hospitaliers de l'Hôpital Jamôt de Yaoundé avant leur récupération (incinérateur).

La gestion des déchets solides de l'Hôpital de District de Biyem-Assi, est assurée par une UHH. L'hôpital possède de plan clair concernant l'hygiène, il ne dispose pas d'un local où les déchets sont stockés avant leur récupération par SECA-HYSACAM. Les DAOM sont stockés dans des bacs fermés de $1 \mathrm{~m}^{3}$ près des toilettes, par contre les D1ASRI sont déposés dans des sacs plastiques noirs près de l'ancien incinérateur (Fig.5). Les DH, une fois évacués des unités, sont censés être stockés d'une façon hygiénique et sécuritaire c'est à dire au niveau d'un local avec une double chambre séparée, aéré, facilement nettoyable, muni d'un point d'eau et d'une serrure. Les services étudiés au niveau de l'hôpital ne possèdent pas de local spécifique pour le stockage intermédiaire. Les déchets avant leur évacuation des services de soins, sont mis dans des bacs fermés de 501 dans les couloirs de chaque service. Sur les unités médicales et medico-techniques visités, le tri est respecté, la collecte se fait au niveau de tous les services 1 fois par jour, 7 jours sur 7 par les agents d'entretiens en charge du nettoyage quotidien de chaque service. Le rythme de la collecte des déchets est régulier, l'évacuation des déchets en dehors de l'hôpital se fait en général par l'intermédiaire des camions par SECAHYSACAM.

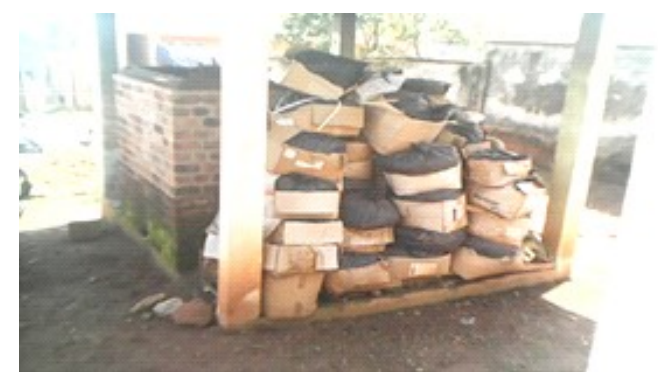

Fig.5. Stockage des déchets solides de l'Hôpital de District de Biyem-Assi avant leur récupération.

\section{Tri}

La pratique du tri précoce des déchets n'est pas répandue dans nos services, $46 \%$ seulement. La qualité de ce tri est mauvaise dans plus d'un tiers des services. C'est au niveau de l'HJY que les erreurs sont les plus fréquentes au cours du tri, engendrant souvent, le mélange de DASRI dans les DAOM Ce qui augmente la quantité de déchets à risque infectieux à traiter et à transporter... : ces erreurs dans le tri résultent d'un manque de formation ou d'information des personnels; de plus, les protocoles expliqués et affichés, de bonnes pratiques, portant sur les procédures à suivre avec les équipements normés, font défaut sur les lieux de travail. 


\section{Conditionnement}

La qualité du conditionnement est aussi fréquemment mauvaise : l'excès de remplissage des sacs de DASRI, le défaut de fermeture, leur présence et leur translation à terre, représentent autant de caractéristiques de pratiques contraires aux normes recommandées, dangereuses pour la sécurité des personnels impliqués, des malades et de l'environnement hospitalier : le tassage ou le compactage en effet interdits lors du conditionnement (et du transport), peuvent aussi se produire lors du transport manuel notifié dans $53 \%$ des cas dans notre enquête. Il faut signaler que la maîtrise de cette étape de la filière d'élimination dépend directement des quantités de DAS produits et sachant qu'à l'état actuel, aucune mesure n'est mise en œuvre pour contrôler et réduire de façon permanente la production de DASRI, les difficultés pour assurer un conditionnement correct et conforme aux normes recommandées, n'iront qu'en s'aggravant dans le temps. En même temps, avec une production incontrôlée de DASRI en particulier, les problématiques sanitaires et de gestion sont plus complexes et beaucoup plus coûteuses.

\section{Stockage intermédiaire}

Rares sont les services cliniques et les unités medico-techniques qui possèdent un en droit spécifique au stockage des déchets; les poubelles pour DAOM sont déposées au niveau du hall ou couloir du service dans d'autres cas. Les DSH, une fois évacués des unités, sont censés être stockés d'une façon hygiénique et sécuritaire c'est à dire au niveau d'un local avec une double chambre séparée, aéré, facilement nettoyable, muni d'un point d'eau et d'une serrure ce qui n'est pas le cas. Les poubelles pour DASRI sont déposées à l'extérieur des chaque pavillons pour l'HJY et des couloirs du service pour l'HDB.

Cette conduite est non hygiénique et non sécuritaire surtout que les poubelles destinées à cet effet contiennent aussi bien les DASRI que les DAOM et qu'elles sont dépourvues de couvercles dans certains cas. Le fait que ces poubelles soient accessibles aux malades et aux visiteurs, constitue un risque traumatique mais surtout infectieux pour eux. En plus de la mauvaise odeur et le développement des insectes.

\section{Traitement des déchets solides hospitaliers}

Les différentes descentes effectuées sur le terrain ont permis de constater que pour les produits de pharmacie, la procédure de destruction des produits pharmaceutiques périmés est maitrisée, cette opération se fait par une commission ad hoc et sanctionnée par un procès-verbal. Les DASRI de l'HJY sont directement traité par l'incinération "in situ": il s'agit d'une installation d'incinération réservée à l'usage d'un établissement de santé et implantée dans son enceinte. Ceux de l'HDB sont traités par l'incinération "centralisée” (régionale): Il s'agit d'une unité extérieure et indépendante des établissements de soins. Cette unité est installée, gérée et exploitée par un organisme indépendant (société privée...). Les DAOM deux hôpitaux sont transport et traités par SECA-HYSACAM par des différentes méthodes d'élimination (enfouissement, décharge et incinération).

\section{Discussion}

Les problèmes liés à la gestion des déchets solides hospitaliers à l'HJY et à l'HDB sont très nombreux, les causes sont dues à au manque de sensibilisation, l'insuffisance de ressources financières et l'insuffisance des ressources matérielles [8-10].

Les responsables des hôpitaux accusent une insuffisance en nombre d'agents d'entretiens, le rythme de la collecte des déchets est irrégulier. Ces résultats montrent que ce rythme est insuffisant pour éviter le risque de développement microbien, surtout en présence d'un climat chaud comme la constaté Daoudi [12-15], d'où la nécessité de collecter ces déchets de manière quotidienne, sinon au plus tard 48 heures après la production. 
Le non-respect du tri des DSH est imputable en grande partie au manque de matériel, à côté du manque de formation et de sensibilisation, comme l'a soulevé la plupart des professionnels de l'Hôpital Jamôt de Yaoundé et de l'Hôpital de District de Biyem-Assi. Les principales raisons justifiant un tri pertinent à la source sont de trois ordres : raisons de sécurité et d'hygiène, raisons économique, raisons techniques [15-18].

Sur le plan du stockage les unités étudiées au niveau de l'Hôpital Jamot de Yaoundé et de l'Hôpital de District de Biyem-Assi ne possèdent pas de local spécifique pour le stockage intermédiaire des déchets avant leur évacuation. Le stockage des DSH au niveau des unités est une mesure contribuant à la sécurité et à l'hygiène de l'unité. C'est pourquoi, il a été préconisé d'avoir un local spécifique pour les déchets, qui doit être aéré, avec un point d'eau, un protocole d'entretien du local et muni d'un système de fermeture.

Les renseignements fournis à l'Hôpital Jamot de Yaoundé et l'Hôpital de District de Biyem-Assi ne nous permettent pas d'évaluer correctement le coût de la gestion des DSH et la part qu'il représente dans le budget global de l'établissement, il est dilué dans le budget d'entretien et d'hygiène, qui souffre toujours d'un manque. Il n'y a pas de programme bien structuré, ni un plan d'action bien établi, les attributions des différents intervenants sont peu claires, les modalités d'une gestion appropriée peu connues, cette activité est reléguée au second plan.

\section{Conclusion}

Au terme du travail mené sur l'évaluation du système de gestion des déchets solides à l'Hôpital Jamot de Yaoundé et l'Hôpital de District de Biyem-Assi. Il ressort de l'analyse des données que les impacts environnementaux sont multiples quand on ne procède pas à une gestion saine des déchets.
Les résultats obtenus pendant cette étude révèlent que, les causes de la mauvaise gestion des déchets solides hospitaliers révèle à $11,73 \%$ que la mauvaise gestion des déchets solides hospitaliers est du eau manque de sensibilisation(A) du personnel et des usagers, à l'insuffisance de ressources financières(B) à 14,28\%.Tandis que, $8,54 \%$ des personnes interviewées parmi le personnel pensent que cette mauvaise gestion est due à l'insuffisance des ressources matérielles(C) appropriées à la gestion des déchets solides. Par contre 11,98\% pensent quelle serait due à $(\mathrm{A}+\mathrm{B})$, $17,67 \%(A+C)$ et $15,45 \%(B+C)$. De même, quelques $20,35 \%$ affirment $(A+B+C)$. A la lumière des résultats obtenus, il ressort que l'organisation de la gestion des déchets solides à l'HJY comme à l'HDB est assurée par un service d'hygiène hospitalier qui est sous la responsabilité d'un technicien du génie sanitaire. Mais fort est de constater que ce services est pratiquement absents à l'HJY, ne répond pas aux attentes du personnel et des usagers. La gestion des déchets solides nécessite inévitablement une implication des responsables à tous les niveaux, une coordination et une collaboration intersectorielle harmonieuse et efficace. Les déchets solides hospitaliers doivent faire l'objet d'une gestion spécifique et rationnelle visant à éviter toute atteinte à la santé de l'homme et à l'environnement [12]. D'où la nécessité de faire une étude au préalable sur les déchets qui sont produits au niveau des hôpitaux ou qui sont susceptibles de l'être, pour déterminer leur quantité et leur typologie et programmer ainsi le matériel et les équipements de conditionnement, de stockage, de transport et de traitement de ces déchets, et le personnel nécessaire pour cette gestion compte tenu entre autres, de la capacité litière occupée de l'hôpital, et du nombre de ses unités.

\section{Références}

Anonyme, 2000. OMS, "Les déchets liés aux soins de santé", aide-mémoire 2 n53; 2000. 
Anonyme, 2003. Directives techniques pour une gestion écologiquement rationnelle des déchets biomédicaux et des déchets de soins médicaux.

Anonyme, 2005a. Plan national de gestion des soins médicaux «manuel d'aide à la décision »P.N.U.E et OMS, pp 3-36.

Anoyme,2005b Gestion des déchets solides d'activités de soins dans les centres de santé primaires « Guide d'aide à la décision ».

Anonyme, 2005c Préparation des Plans Nationaux de Gestion des Déchets de Soins Médicaux en Afrique Subsaharienne "Manuel d'aide à la décision »Secrétariat de la Convention de Bâle Organisation mondiale de la Santé p5-6

Anonyme, 2005d Gestion sûre des déchets hospitaliers. Aide-mémoire pour une stratégie nationale. p. 18

Anonyme, 2010. Déchets et Santé. Observatoire régional de la santé, Nord-Pas-de-Calais.24 p.

Anonyme, 2007a. Assainissement et hygiène dans les pays en voie développement : étude de cas en République Démocratique du Congo, pp-65.

Anonyme, 2007b. Stratégie nationale de gestion des déchets. Draft III. MINEP, Yaoundé 138p

Anonyme, 2008. Plan De Gestion Des DÉCHETS HOSPITALIERS P 6-70

Anonyme, 2010. Déchets et Santé. Observatoire régional de la santé, Nord-Pas-de-Calais. 24 p.

Anonyme, 2011 «Manuel de gestion des déchets médicaux ».23p

Anonyme 2012, Cameroun tribune «Le décret No2012/2809/PM du 26 septembre2012 relatif aux conditions de tri, de collecte, de stockage, de transport, de récupération, de recyclage, de traitement et d'élimination finale des déchets »Septembre N 10195 pp 19-21

Ashmore J., Atsumi S. \&Badawy L., 2008. Le toolkit de gestion de camp. Conseil Norvégien pour les réfugiés, Oslo, Norvège. 594 p

Abdelsadok. N., 2010. Etude d'accompagnement pour la gestion des déchets médicaux au Maroc, pp34-46.

Kane A et al. Bulletin de l'Organisation mondiale de la Santé, Recueil d'articles $N^{\circ}$ 2, pp 44-49, 2000 .

Daoudi, 2008. Evaluation de la gestion des déchets solides médicaux et pharmaceutiques à hôpital Hassan II d'Agadir, pp10-25.

Deoux, P. \& Deoux, S. 1993. L'écologie c'est la santé : l'impact des nuisances de l'environnement sur la santé. Connaitre pour agir au quotidien. Frison-Roche pub. paris 492p 